\title{
OPTIMIZATION AND PRACTICAL IMPLEMENTATION OF ULTRAFAST 2D NMR EXPERIMENTS
}

Luiz H. K. Queiroz Júnior*

Departamento de Química, Universidade Federal de São Carlos, Rod. Washington Luís, km 235, 13565-905 São Carlos - SP / Instituto de Química, Universidade Federal de Goiás, CP 131, 74001-970 Goiânia - GO, Brasil

Antonio G. Ferreira

Departamento de Química, Universidade Federal de São Carlos, Rod. Washington Luís, km 235, 13565-905 São Carlos - SP, Brasil Patrick Giraudeau

Université de Nantes, CNRS, Chimie et Interdisciplinarité: Synthèse, Analyse, Modélisation UMR 6230, B.P. 92208, 2 rue de la Houssinière, F-44322 Nantes Cedex 03, France

Recebido em 30/4/12; aceito em 17/10/12; publicado na web em 8/3/13

\begin{abstract}
Ultrafast 2D NMR is a powerful methodology that allows recording of a 2D NMR spectrum in a fraction of second. However, due to the numerous non-conventional parameters involved in this methodology its implementation is no trivial task. Here, an optimized experimental protocol is carefully described to ensure efficient implementation of ultrafast NMR. The ultrafast spectra resulting from this implementation are presented based on the example of two widely used 2D NMR experiments, COSY and HSQC, obtained in $0.2 \mathrm{~s}$ and $41 \mathrm{~s}$, respectively.
\end{abstract}

Keywords: ultrafast 2D NMR; COSY; HSQC.

\section{INTRODUCTION}

Analysis of the historical panorama of Nuclear Magnetic Resonance (NMR) reveals great evolution of this phenomenon ${ }^{1-3}$ since the first records. Part of this impressive evolution is due the emergence of two-dimensional (2D) ${ }^{4,5}$ and imaging ${ }^{6,7}$ experiments. In spite of this progress, conventional multidimensional NMR experiments still require a high number of scans to sample the indirect $t_{1}$ domain, leading to long experiment durations (from several minutes to several hours). This limitation creates not only timetable constraints, but also renders conventional $\mathrm{nD}$ NMR experiments unsuitable for the study of kinetic or dynamic phenomena occurring on a short timescale or for hyphenated techniques such as LC-NMR. Several methodologies have been proposed to overcome this limitation in order to obtain spectra in shorter time frames, ${ }^{8-12}$ most notably the technique proposed by Frydman et al., ${ }^{13}$ also known as "ultrafast NMR" (UF-NMR). This technique allows performing of multidimensional experiments in a single scan, provided that sensitivity is sufficient.

The approach of UF-NMR differs to that used in its conventional counterpart, in terms of spin evolution and data acquisition. In a conventional 2D NMR experiment, spins are uniformly excited and $t_{1}$ evolution is systematically incremented in a series of distinct experiments. Acquisition is carried out by the storage of different FIDs (Free Induction Decays) from the corresponding incremented experiments. ${ }^{5}$ In the UF-NMR approach, the usual $t_{1}$ encoding is replaced by spatial encoding, and after a conventional mixing period, the spatially encoded information is decoded by a detection block based on echo planar imaging (EPI). ${ }^{7}$ The principles of UF-NMR have been extensively described in recent reviews ${ }^{14-16}$ and are summarized below.

The spatial encoding scheme initially proposed by Frydman ${ }^{13,17}$ relies on a succession of frequency-selective pulses applied during alternating bipolar gradient pairs. However, it suffers from several drawbacks as it requires fast gradient switching, carefully synchronized with RF irradiation. Moreover, it leads to the appearance of undesirable "ghost peaks"18 in the indirect domain. Consequently,

*e-mail: professorkeng@gmail.com this discrete excitation scheme was replaced by several continuous encoding patterns ${ }^{19-22}$ relying on the combination of continuous frequency swept pulses applied during a bipolar gradient. The most efficient approach in terms of resolution and sensitivity is probably that proposed by Pelupessy (Figure 1S, supplementary material), ${ }^{20}$ where spatial encoding is carried out in a constant-time manner through the application of two chirp pulses concomitantly with a bipolar gradient pair $\left(G_{\mathrm{e}}\right)$. The first pulse/gradient combination results in quadratic dephasing $\left(z^{2}\right)$ which requires the application of $z^{2}$ gradients for refocusing of the magnetizations arising from different $z$ positions. An alternative consists of applying, following the first pulse, an identical pulse together with an opposite gradient. This leads to linear dephasing that depends linearly on position along the $\mathrm{z}$ axis and resonance frequency $\left(\mathrm{W}_{1}\right)$. It should also be noted that in this excitation scheme, spatial encoding is performed in a constant-time manner. Briefly, we can imagine the sample being divided into infinitesimal slices with dephasing varying linearly according to position.

The mixing step remains exactly the same as that used in conventional experiments, ${ }^{13,20}$ preserving the linear dephasing obtained by the previous encoding block. For acquisition, a scheme based on echo planar imaging (EPI $)^{7}$ is used. Firstly, after the receiver is open, the acquisition gradient $\mathrm{G}_{\mathrm{a}}$ (with duration of a few hundred microseconds) is applied to refocus the dephasing acquired in the course of spatial encoding, leading to the formation of a series of echoes whose positions are proportional to the resonance frequencies that have been encoded (Figure 2S-a, supplementary material), where the signals observed during this period are similar to an $1 \mathrm{D}$ spectrum. As a consequence, the first dimension of the 2D spectrum, also called the "ultrafast dimension", is obtained without any Fourier Transform.

In order to obtain the second dimension, alternated $\pm \mathrm{G}_{\mathrm{a}}$ gradients (Figure 2S-b, supplementary material) are applied, typically during one hundred milliseconds, to monitor the spin evolution in the course of the $t_{2}$ acquisition time under the effect of classical parameters: resonance frequency, transverse relaxation, $J$-couplings, etc. These alternated gradients induce a series of refocusings and defocusings, which results in a set of symmetrical 1D spectra (mirror-image echoes). As the conventional interactions are characteristic of this 
dimension, it is generally called a "conventional dimension". After data rearrangement and FT along $t_{2}$, a 2D spectrum is obtained containing the same information as its conventional counterpart.

Many studies have been performed to improve the performances of this promising technique, in terms of sensitivity, ${ }^{23}$ resolution, ${ }^{24,25}$ lineshape ${ }^{26}$ and accessible spectral width. ${ }^{26,27}$ Following these improvements, UF-NMR has been applied successfully in a broad range of domains, including the study of mechanistic, ${ }^{28}$ kinetic $^{29}$ or dynamic ${ }^{30}$ processes, the coupling with $e x$-situ Dynamic Nuclear Polarisation ${ }^{31}$ or the application to quantitative analysis. ${ }^{32}$ Nevertheless, due to the numerous non-conventional parameters involved in this methodology and to the specific processing required, its implementation is no trivial task. In this context, the aim of the present paper was to describe the practical protocol used in the implementation of this technique to record UF-NMR spectra. The main difficulties regarding the implementation of this powerful tool are described, based on the example of two widely used 2D NMR experiments: COSY and HSQC.

\section{EXPERIMENTAL}

\section{Sample preparation}

For the initial calibration of encoding gradients and pulses, a $600 \mu \mathrm{L}$ solution of $\mathrm{H}_{2} \mathrm{O} / \mathrm{D}_{2} \mathrm{O}(90: 10)$ was prepared. For the conventional and ultrafast COSY experiments, Levamisol (purchased from Sigma-Aldrich) solution was obtained by dissolving $10 \mathrm{mg}$ of this compound in $600 \mu \mathrm{L}$ of MeOD. The concentrated solution used for the conventional and ultrafast HSQC experiments was prepared by mixing $200 \mu \mathrm{L}$ of 1 -bromohexane in $400 \mu \mathrm{L}$ of $\mathrm{CDCl}_{3}$. All these samples were filtered and transferred to $5 \mathrm{~mm}$ tubes. Deuterated solvents were purchased from Cambridge Isotope Laboratories, Inc.

\section{General NMR parameters}

All the NMR experiments were performed at $298 \mathrm{~K}$ on a Bruker Avance III 400 spectrometer, operating at a frequency of 400.15 $\mathrm{MHz}{ }^{1} \mathrm{H}$, with a $5 \mathrm{~mm}$ SmartProbe ${ }^{\circledR}$ with gradients along the $z$-axis $(50 \mathrm{G} / \mathrm{cm}$ for $100 \%$ gradient amplitude). For both conventional and ultrafast experiments, the pulse width for $\pi / 2$ pulse was $8.13 \mathrm{~ms}$ for ${ }^{1} \mathrm{H}$ and $15.0 \mu$ s for ${ }^{13} \mathrm{C}$.

\section{Ultrafast 2D NMR parameters}

The main acquisition parameters for calibration of encoding gradients were set as follows: time domain size - 2984 data points (real + imaginary); spectral width - 300 ppm; acquisition time - 10 ms; number of scans - 1 ; central frequency $-\mathrm{H}_{2} \mathrm{O}$ peak; duration of refocusing gradient $\left(G_{1}\right)-10 \mathrm{~ms}$; delay between $\pi$ pulse and $G_{1}-1$ ms; acquisition gradient delay $\left(d_{a g}\right)-5$ ms; $G_{1}$ strength $-4 \mathrm{G} / \mathrm{cm}$; hard pulse duration - calibrated as for the conventional experiments. The $\mathrm{d}_{\mathrm{ag}}$ delay was adjusted to place the echo in the center of the acquisition window. For processing, an apodization function was applied with LB (Lorentzian-Broadening) of -20 Hz and GB (Gaussian-Broadening) at the position of the echo, i.e. 0.5. A Fourier transform (FT) was then done, followed by phase correction. This last step is crucial because of the phase dispersion induced by the gradients, therefore it was first performed by first order phase correction (typically $70000^{\circ}$ ), and finally by a small zero order correction to obtain a symmetric image.

For the calibration of encoding pulses, an adiabatic pulse with a smoothed chirp shape was created ( $10 \%$ smoothing), with a frequency range of $60 \mathrm{kHz}, 10 \%$ higher than the frequency dispersion induced by the $6.77 \mathrm{G} / \mathrm{cm}$ excitation gradient $\left(\mathrm{G}_{\mathrm{e}}\right)$, and with $15 \mathrm{~ms}$ duration.
The shaped pulse power level was calibrated starting from $0.1 \mathrm{~mW}$. The optimum value for the shaped pulse power level was $0.32 \mathrm{~W}$.

To perform the UF-COSY experiment, the acquisition parameters set up for the encoding block were identical to those calibrated previously. The coherence selection gradients strength was $45 \mathrm{G} /$ $\mathrm{cm}$ during $1000 \mathrm{~ms}$, and the purge gradient strength was $-10 \mathrm{G} / \mathrm{cm}$ with $400 \mathrm{~ms}$ duration. The acquisition times for direct and indirect dimensions were 65.59 and $0.12 \mathrm{~ms}$, respectively. For the acquisition of 35 and $-35.008 \mathrm{G} / \mathrm{cm}$ (to compensate for shearing effects), gradients were applied for $236 \mathrm{~ms}$ each, with a gradient rise time of $20 \mu$ s (recovery delay in pulse sequence).

For the UF-HSQC experiment, identical chirp pulses were used but applied in the presence of $\pm 20 \mathrm{G} / \mathrm{cm}$ encoding gradients to account for the larger ${ }^{13} \mathrm{C}$ frequency range. The acquisition times for direct and indirect dimensions were 65.59 and $0.02 \mathrm{~ms}$, respectively. Acquisition gradients were identical to those employed for UF-COSY. The INEPT delay was set to $1.72 \mathrm{~ms}$. Eight scans were recorded for sensitivity and phase-cycling purposes.

It is important to highlight that all hard pulses are calibrated in exactly the same way as for the conventional experiments.

Ultrafast spectra processing was performed by using an in-house routine in TopSpin ${ }^{\circledR}$. This routine included conventional zero-filling and apodization features, including apodization in the ultrafast dimension $(\mathrm{LB}=-50 \mathrm{~Hz}$ and $\mathrm{GM}=0.5)$ designed to optimize line shapes and sensitivity in the spatially-encoded dimension, ${ }^{25}$ and also a Sine apodization function in the conventional dimension $(\mathrm{LB}=-8 \mathrm{~Hz}$ and $\mathrm{GM}=0.08)$. Spectra were processed in magnitude mode.

The UF-COSY and UF-HSQC pulse sequence (Bruker format) are available in the supplementary material. The processing routine is freely available on demand.

\section{Conventional 2D NMR parameters}

The conventional $\mathrm{COSY}^{33}$ and $\mathrm{HSQC}^{34} 2 \mathrm{D}$ experiments were recorded using routine pulse sequence available in the Bruker TopSpin 3.1 software. The conventional COSY spectrum was performed in 6 scans, using 4096 data points (real + imaginary), $128 t_{1}$ increments and a $2 \mathrm{~s}$ recovery delay. For the conventional HSQC, the number of $t_{1}$ increments was 128 , with a recovery delay of $3 \mathrm{~s}, 4096$ data points (real + imaginary) and a total of 8 scans.

\section{RESULTS AND DISCUSSION}

\section{Calibration of encoding gradients and pulses}

Spatially selective excitation is a fundamental step of UF-NMR experiments which needs to be carefully calibrated. Figure 1 describes the pulse sequence designed for this preliminary calibration. The first

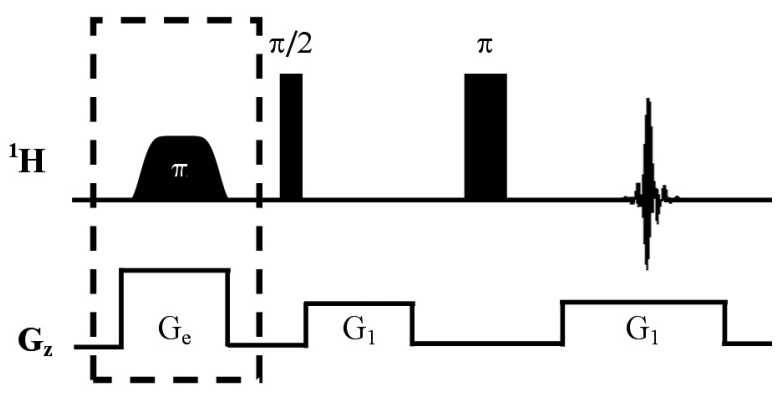

Figure 1. Pulse sequence for calibration of the gradients needed for ultrafast $2 D$ NMR experiments. The extra dashed block is appended to calibrate the chirp pulses needed for spatial encoding. Other experimental details, needed to implement this experiment, are included in the experimental section 
step consists of calibrating the excitation pulses in order to ensure that their frequency width matches the frequency dispersion induced by the excitation gradients $\left(\mathrm{G}_{\mathrm{e}}\right)$.

A sample with only one resonance frequency (here $\mathrm{H}_{2} \mathrm{O}$ in $\mathrm{D}_{2} \mathrm{O}$ ) should be used for more efficient calibration. First, the dashed block (pulse calibration stage) is inactivated by setting the $G_{e}$ strength to $0.0 \mathrm{G} / \mathrm{cm}$ and the selective pulse power level to $0.0 \mathrm{~W}$. The rest of the sequence generates a spin echo which, after Fourier transform, yields the excitation profile of the sample (Figure 2a). Because of the phase dispersion induced by the gradients, a large first-order phase correction followed by a small zero order correction, are necessary to obtain a symmetric image.

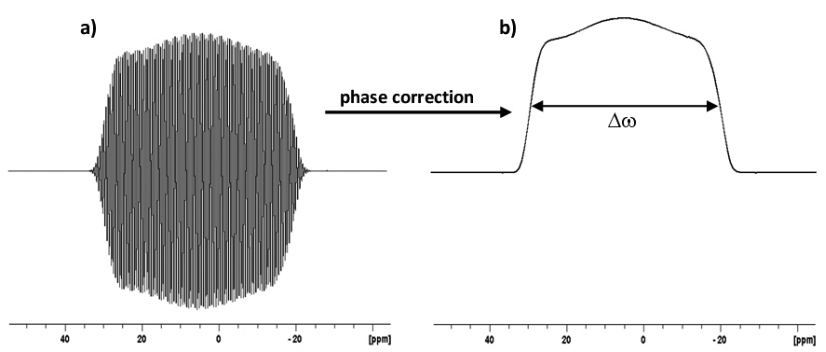

Figure 2. Image of the excitation profile obtained from the pulse sequence in Figure 1 (without the extra block), allowing for gradient strength calibration. Without ( $a$ ) and with (b) phase correction. The frequency dispersion D $\omega$ induced by $G_{2}$ is $\gamma \cdot G_{2} \cdot L$, where $\gamma$ is the gyromagnetic ratio of detected nuclei and $L$ the height of the detection coil. For both spectra, the acquisition time, number of data points and number of scans were $12.58 \mathrm{~ms}, 2984$ and 1, respectively. For the processing parameters, the window function was the Gaussian multiplication with line broadening of -20.0 and Gaussian maximum position of 0.5 for both spectra, while the phase corrections of zero and first order were set to zero for spectrum $2 a$ and to $11 \times 10^{6}$ and $-23 \times 10^{6}$ for spectrum $2 b$

The frequency dispersion induced by $\mathrm{G}_{1}$ corresponds to the width of the excitation profile (Figure $2 b$ ). In order to perform ultrafast experiments, the gradient strength should be set to induce a frequency dispersion much higher than the chemical shift range, in order to ensure that all resonance frequencies are equally addressed at a given $z$ position. Here, a $6.77 \mathrm{G} / \mathrm{cm}$ gradient is chosen, leading to a $54 \mathrm{kHz}$ frequency dispersion, much higher than the usual ${ }^{1} \mathrm{H}$ spectral range. The next step consists of calibrating the adiabatic pulse used for spatial encoding. As discussed by Pelupessy, ${ }^{20}$ its bandwidth should be slightly higher than the frequency dispersion induced by the gradients, to ensure that the whole sample is excited. The pulse calibration stage (Figure 1 - dashed block) is then activated, and the shaped pulse power level is decreased parametrically until complete inversion of the excitation profile is obtained (Figure 3S, supplementary material). In our case the optimum shaped pulse power level was $0.32 \mathrm{~W}$. This gradient calibration procedure need be performed only once, so the optimized parameters can be used for other experiments.

\section{UF-COSY 2D implementation}

With both shaped pulse and encoding gradient properly calibrated, we proceed to the implementation of a homonuclear bidimensional NMR experiment: Ultrafast COSY (UF-COSY). The pulse sequence used is shown in Figure 4S, supplementary material. For the aforementioned reasons, the constant-time spatial encoding proposed by Pelupessy ${ }^{20}$ was chosen to perform our experiments.

The UF-COSY pulse sequence starts with a nonselective $\pi / 2$ pulse, followed by constant-time spatial encoding in which two adiabatic pulses are applied simultaneously with a bipolar excitation gradient pair $\left(\mathrm{G}_{\mathrm{e}}\right)$, leading to a spatially selective encoding of the spins. As the phase has a quadratic dependence on $z^{2}$ after the first chirp pulse, ${ }^{20}$ it is necessary to apply a second pulse with an opposite gradient, to remove this effect and make the $z$-positions of the spins directly proportional to their resonance frequency. The mixing step is the same as for a conventional experiment, including coherence-selection gradients. A purge gradient $\left(\mathrm{G}_{\mathrm{p}}\right)$ is used to shift the signal positions in the ultrafast dimension, in order to center them in the observation window. Finally, a bipolar acquisition gradient pair $\left(G_{a}\right)$ is applied during $2 . \mathrm{T}_{\mathrm{a}}$, to refocus the dephasing induced by the spatial encoding step, resulting in the formation of successive echoes according to the distinct precession frequency of the nuclei. This acquisition scheme is repeated $\mathrm{N}_{2}$ times leading to complete sampling of the $\left(k, t_{2}\right)$ space in a single scan. The acquisition gradient amplitude $\mathrm{G}_{\mathrm{a}}$ should be chosen to ensure that the desired spectral width is observed in the spatially-encoded dimension. As a consequence, the maximum observable spectral width is limited by the maximum gradient amplitude available. While choosing $\mathrm{G}_{\mathrm{a}}=100 \%$ of the maximum gradient amplitude would ensure the observation of the largest spectral range possible, we recommend instead to first set $\mathrm{G}_{\mathrm{a}}=70 \%$ to avoid reaching the limits of the gradient hardware, principally to preserve its integrity but also because instabilities in the bipolar acquisition gradient train are observed for high $\mathrm{G}_{\mathrm{a}}$ values. $\mathrm{G}_{\mathrm{a}}$ can then be adjusted more finely depending on the spectral range to be detected.

Careful attention must be paid to shearing effects in the course of the acquisition process. Because of possible gradient offset, the positive acquisition gradient may not be the exact opposite of the negative gradient. This effect creates a linear shifting of the peak position during acquisition, leading to large resolution losses in the 2D spectrum. Therefore, fine tuning of the negative gradient amplitude must be performed to obtain optimum spectra.

An example of UF-COSY 2D spectrum is given in Figure $3 \mathrm{~b}$ for a Levamisol sample. The spectrum is recorded in $0.17 \mathrm{~s}$, whereas 30 min are necessary to record its conventional counterpart (Figure 3a). The two spectra exhibit the same homonuclear correlations, thus proving the efficiency of the ultrafast approach.

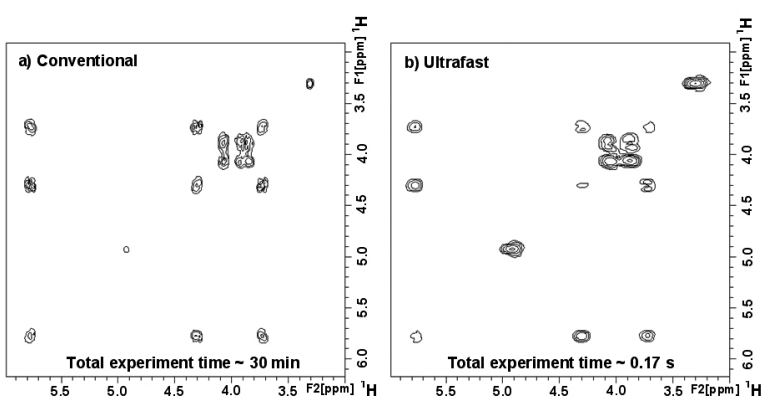

Figure 3. (a) Conventional and (b) ultrafast COSY spectrum obtained on a $0.08 \mathrm{~mol} \mathrm{~L}^{-1}$ Levamisol sample in methanol, on a $400 \mathrm{MHz}$ spectrometer at $298 \mathrm{~K}$. The conventional spectrum was recorded with acquisition times of $365.96 \mathrm{~ms}$ (direct) and $11.43 \mathrm{~ms}$ (indirect), 4096 data points, 6 scans and $128 t_{1}$ increments, and was processed using the sine-squared function with sine bell shift equals to 3 in both dimensions. The ultrafast spectrum was recorded in a single scan

\section{UF-HSQC 2D implementation}

The pulse sequence for implementation of the heteronuclear $2 \mathrm{D}$ HSQC experiment (UF-HSQC) is described in Figure 5S, supplementary material. The spatial encoding stage was performed on the ${ }^{13} \mathrm{C}$ channel, therefore higher encoding gradients $\pm \mathrm{G}_{\mathrm{e}}$ were applied as described in the experimental part in order to account for the larger ${ }^{13} \mathrm{C}$ spectral range. 
This UF-HSQC sequence has many steps in common with a conventional HSQC experiment. Initially, an INEPT block allows improving of the sensitivity of the ${ }^{13} \mathrm{C}$ nuclei by polarization transfer from the ${ }^{1} \mathrm{H}$ nuclei. Subsequently, spatial encoding is performed on the ${ }^{13} \mathrm{C}$ channel to permit the position-dependent evolution of the ${ }^{13} \mathrm{C}$ nuclei, and in the middle of this period, a $\pi$ pulse is applied on the ${ }^{1} \mathrm{H}$ channel to refocus $J_{\mathrm{CH}}$ couplings. After a retro-INEPT block, the signal is detected on the ${ }^{1} \mathrm{H}$ channel. The echoes observed during the first acquisition gradient correspond to the ${ }^{13} \mathrm{C}$ spectrum, while the ${ }^{1} \mathrm{H}$ spectrum is obtained from the EPI dimension. Additionally, $\pi$ pulses are added on the ${ }^{13} \mathrm{C}$ channel between acquisition gradients to perform ${ }^{13} \mathrm{C}$ decoupling.

As the ultrafast dimension window is limited by the strength of acquisition gradients, the spectral width observable in the ${ }^{13} \mathrm{C}$ dimension is limited to a few tens of ppm. ${ }^{27}$ In order to overcome this limitation, the recently proposed "gradient-folding" method ${ }^{27}$ to improve the spectral width of ultrafast 2D NMR experiments was used. The function of the gradients $G_{1}$ and $G_{2}$ is to enable the folding of the peaks that are outside the spectral window in the ultrafast dimension.

The spectrum obtained after performing this UF-HSQC pulse sequence on a 1-bromohexane sample is shown in Figure $4 \mathrm{~b}$. Eight transients were accumulated for the sake of sensitivity and to perform the conventional HSQC phase cycling for a more efficient coherence selection. However, the whole 2D spectrum is recorded in less than 1 minute, showing the same information as disclosed on the conventional spectrum (Figure 4a), but in a much shorter timeframe. The highlighted signal $(13.9 \mathrm{ppm})$ in the ultrafast spectrum is folded in the ultrafast dimension by adjusting $\mathrm{G}_{1}(30 \mathrm{G} / \mathrm{cm})$ and $\mathrm{G}_{2}$ $(7.5 \mathrm{G} / \mathrm{cm})$ gradients.

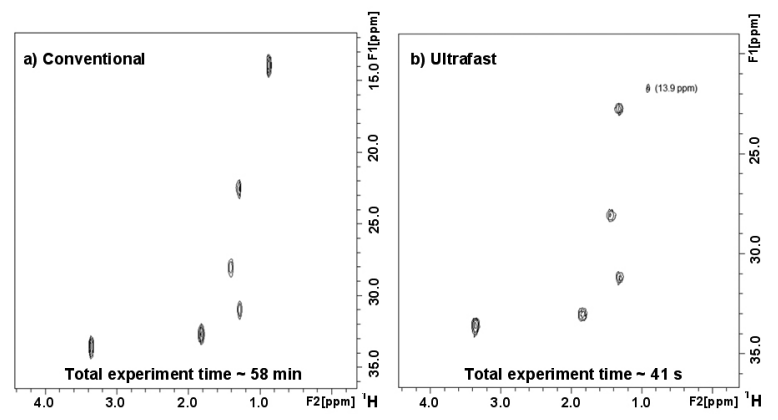

Figure 4. (a) Conventional and (b) ultrafast HSQC spectrum obtained on a $2.37 \mathrm{~mol} \mathrm{~L}^{-1}$ 1-bromohexane sample in chloroform, on a $400 \mathrm{MHz}$ spectrometer at $298 \mathrm{~K}$. The conventional spectrum was recorded with acquisition times of $365.96 \mathrm{~ms}$ (direct) and $2.62 \mathrm{~ms}$ (indirect), 4096 data points, 8 scans and 128 $t_{1}$ increments, and was processed using the sine-squared function with sine bell shift equals to 2 in both dimensions. The ultrafast spectrum was recorded in 8 scans and there is a folded peak (13.9 ppm) indicated on this spectrum in order to overcome the aforementioned limitation of ultrafast spectral window

\section{CONCLUSIONS}

This paper describes a detailed protocol for implementing both homo- and heteronuclear multidimensional ultrafast experiments. In spite of its impressive performance, it should be noted that ultrafast NMR is characterized by several limitations and compromises which potentially affect its performance. In particular, a trade-off has to be made between resolution, sensitivity and spectral width, as described in recent papers. ${ }^{23,27,35}$ However, these limitations are now well understood, and solutions have been proposed to overcome them. These solutions include, for example, the use of multi-echo excitation to limit sensitivity losses due to molecular diffusion ${ }^{24}$ or the use of $k$-space folding strategies to increase the accessible spectral width..$^{27,35}$
The sensitivity issues of ultrafast 2D NMR are also an important point worth discussing. The sensitivity of a single-scan experiment is of course lower than that of a conventional experiment where signal is accumulated for minutes or hours. This single-scan sensitivity is clearly highly dependent on the spectrometer and probe, but typically $100 \mathrm{mM}$ samples are used in $400 \mathrm{MHz}$ instruments equipped with conventional probes, while $10 \mathrm{mM}$ samples are commonly used in $500 \mathrm{MHz}$ instruments equipped with cryogenic probes. ${ }^{36}$ These values seem quite high, but lower concentrations are easily reached by signal averaging, a "multi-scan single shot" approach whose potentialities have been recently demonstrated. ${ }^{37}$ Following these developments, ultrafast NMR has been recently applied to a variety of situations, such as monitoring of fast organic reactions, ${ }^{28,38}$ the study of biologically relevant samples, ${ }^{30,38}$ coupling with hyphenated techniques ${ }^{39}$ and measurement of residual dipolar couplings in oriented media. ${ }^{40}$

We are currently applying these experiments to the study of dynamic processes occurring on a very short timescale that could not hitherto be followed by any other kind of 2D NMR experiment.

\section{SUPPLEMENTARY MATERIAL}

Free access in PDF format available on-line at http://quimicanova. sbq.org.br.

\section{ACKNOWLEDGEMENTS}

The authors are grateful to Prof. S. Akoka for discussions. P. Giraudeau acknowledges funding from the "Agence Nationale de la Recherche" (ANR Grant 2010-JCJC-0804-01). L. H. K. Queiroz Jr. and A. G. Ferreira acknowledge the Coordenação de Aperfeiçoamento de Pessoal de Nível Superior (CAPES), the Conselho Nacional de Desenvolvimento Científico e Tecnológico (CNPq) and the Fundação de Amparo à Pesquisa do Estado de São Paulo (FAPESP) for the financial support.

\section{REFERENCES}

1. Rabi, I.; Zacharias, J.; Millman, S.; Kusch, P.; Phys. Rev. 1938, 53, 318.

2. Bloch, F.; Hansen, W.; Packard, M.; Phys. Rev. 1946, 69, 127.

3. Purcell, E.; Torrey, H.; Pound, R.; Phys. Rev. 1946, 69, 37.

4. Jeener, J.; International Ampere Summer School II, Basko Polje, Yugoslavia, 1971.

5. Aue, W. P.; Karhan, J.; Ernst, R. R.; J. Chem. Phys. 1976, 64, 2229.

6. Lauterbur, P. C.; Nature 1973, 242, 190.

7. Mansfield, P.; Maudsley, A. A.; J. Phys. C: Solid State Phys. 1976, 9, L409.

8. Mandelshtam, V.; Taylor, H.; Shaka, A.; J. Magn. Reson. 1998, 133, 304.

9. Kupče, E..; Freeman, R.; J. Magn. Reson. 2003, 162, 300.

10. Brüschweiler, R.; Zhang, F.; J. Chem. Phys. 2004, 120, 5253.

11. Schanda, P.; Brutscher, B.; J. Am. Chem. Soc. 2005, 127, 8014.

12. Vitorge, B.; Bodenhausen, G.; Pelupessy, P.; J. Magn. Reson. 2010, 207, 149.

13. Frydman, L.; Scherf, T.; Lupulescu, A.; Proc. Natl. Acad. Sci. 2002, 99, 15858.

14. Gal, M.; Frydman, L. In Encyclopedia of NMR; Grant, D. M.; Harris, R. K., eds.; Wiley: New York, 2009, vol. 10.

15. Mishkovsky, M.; Frydman, L.; Annu. Rev. Phys. Chem. 2009, 60, 429.

16. Tal, A.; Frydman, L.; Prog. Nuc. Magn. Res. Spec. 2010, 57, 241.

17. Frydman, L.; Lupulescu, A.; Scherf, T.; J. Am. Chem. Soc. 2003, 125, 9204.

18. Shrot, Y.; Frydman, L.; J. Magn. Reson. 2003, 164, 351.

19. Shrot, Y.; Shapira, B.; Frydman, L.; J. Magn. Reson. 2004, 171, 163.

20. Pelupessy, P.; J. Am. Chem. Soc. 2003, 125, 12345. 
21. Tal, A.; Shapira, B.; Frydman, L.; J. Magn. Reson. 2005, 176, 107.

22. Shrot, Y.; Frydman, L.; J. Chem. Phys. 2008, 128, 052209.

23. Giraudeau, P.; Akoka, S.; J. Magn. Reson. 2008, 192, 151.

24. Giraudeau, P.; Akoka, S.; J. Magn. Reson. 2008, 190, 339.

25. Pelupessy, P.; Duma, L.; Bodenhausen, G.; J. Magn. Reson. 2008, 194. 169.

26. Giraudeau, P.; Akoka, S.; Magn. Reson. Chem. 2011, 49, 307.

27. Giraudeau, P.; Akoka, S.; J. Magn. Reson. 2010, 205, 171.

28. Queiroz Jr, L. H. K.; Giraudeau, P.; dos Santos, F. A.; de Oliveira, K. T.; Ferreira, A. G.; Magn. Reson. Chem. 2012, 50, 496.

29. Giraudeau, P.; Lemeunier, P.; Coutand, M.; Doux, J.-M.; Gilbert, A.; Remaud, G. S.; Akoka, S.; J. Spectrosc. Dyn. 2011, 1, 2.

30. Lee, M.-K.; Gal, M.; Frydman, L.; Varani, G.; Proc. Natl. Acad. Sci. 2010, 107, 9192

31. Frydman, L.; Blazina, D.; Nat. Phys. 2007, 3, 415.
32. Giraudeau, P.; Remaud, S.; Akoka, S.; Anal. Chem. 2009, 81, 479.

33. Nagayama, K.; Kumar, A.; Wüthrich, K.; Ernst, R. R.; J. Magn. Reson. 1980, 40,321

34. Bodenhausen, G.; Ruben, D. J.; Chem. Phys. Lett. 1980, 69, 185.

35. Shrot, Y.; Frydman, L.; J. Chem. Phys. 2009, 131, 224516.

36. Giraudeau, P.; Massou, S.; Robin, Y.; Cahoreau, E.; Portais, J-C.; Akoka, S.; Anal. Chem. 2011, 83, 3112.

37. Pathan, M.; Akoka, S.; Tea, I.; Charrier, B.; Giraudeau, P.; Analyst 2011, 136,3157

38. Pardo, Z. D.; Olsen, G. L.; Fernández-Valle, M. E.; Frydman, L.; Martínez-Álvarez, R.; Herrera, A.; J. Am. Chem. Soc. 2012, 134, 2706.

39. Queiroz Jr, L. H. K.; Queiroz, D. P. K.; Dhooghe, L.; Ferreira, A. G.; Giraudeau, P.; Analyst 2012, 137, 2357.

40. Giraudeau, P.; Montag, T.; Charrier, B.; Thiele, C. M.; Magn. Reson. Chem. 2012, 50, S53. 


\section{OPTIMIZATION AND PRACTICAL IMPLEMENTATION OF ULTRAFAST 2D NMR EXPERIMENTS}

Luiz H. K. Queiroz Júnior*

Departamento de Química, Universidade Federal de São Carlos, Rod. Washington Luís, km 235, 13565-905 São Carlos - SP / Instituto de Química, Universidade Federal de Goiás, CP 131, 74001-970 Goiânia - GO, Brasil

Antonio G. Ferreira

Departamento de Química, Universidade Federal de São Carlos, Rod. Washington Luís, km 235, 13565-905 São Carlos - SP, Brasil Patrick Giraudeau

Université de Nantes, CNRS, Chimie et Interdisciplinarité: Synthèse, Analyse, Modélisation UMR 6230, B.P. 92208,2 rue de la Houssinière, F-44322 Nantes Cedex 03, France
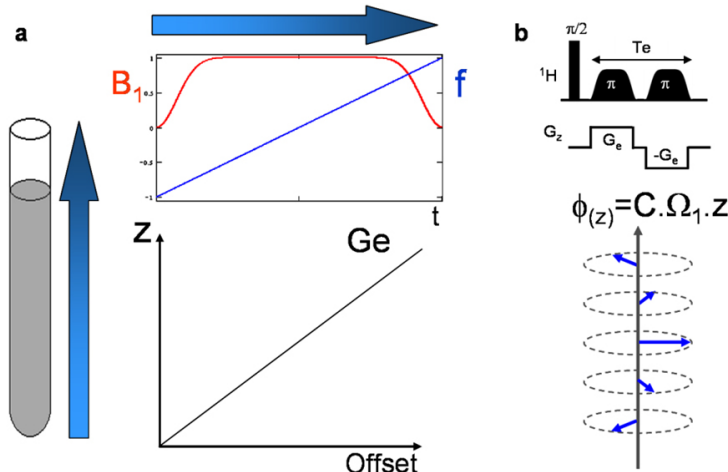

Figure 1S. (a) Graphical representation of continuous spatial encoding, in which a chirp pulse is applied concomitantly to a gradient pulse $\left(G_{e}\right)$ along $z$ axis. (b) The scheme proposed by Pelupessy ${ }^{20}$ comprises the application of a $90^{\circ}$ hard pulse followed by two $\pi$ chirp pulses applied together with a bipolar pair of gradients

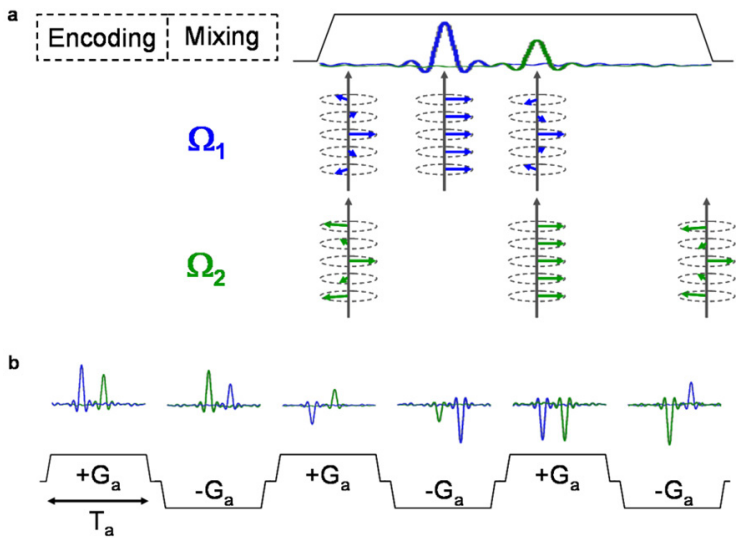

Figure 2S. (a) Representation of the ultrafast dimension acquisition by the application of a gradient pulse, in order to remove the dephasing created during the spatial encoding step. As result, the echo peaks are formed as the dephasing is being refocused. (b) Representation of the conventional dimension acquisition by the use of a bipolar pair of gradient pulses, which results in the monitoring of conventional parameters evolution as a series of sub-spectra are being collected

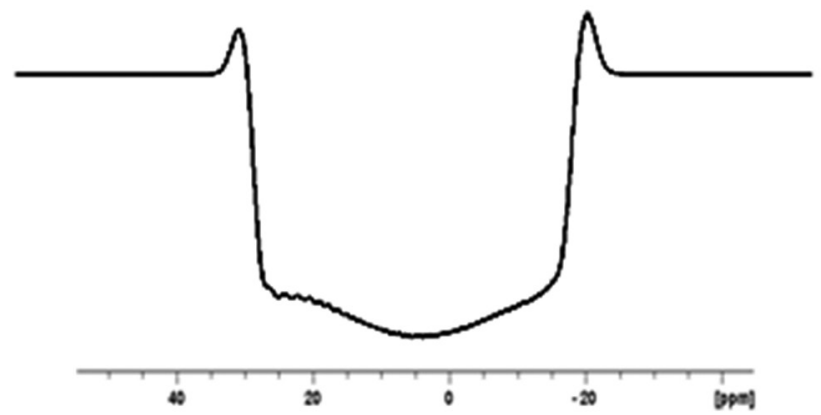

Figure 3S. Image of the excitation profile obtained from the pulse sequence in Figure 1 (with the extra block) and after phase correction, allowing for the chirp pulse power calibration before performing ultrafast experiments. The acquisition and processing parameters are the same mentioned for the Figure $2 b$, unless the chirp pulse power that was $0.32 \mathrm{~W}$

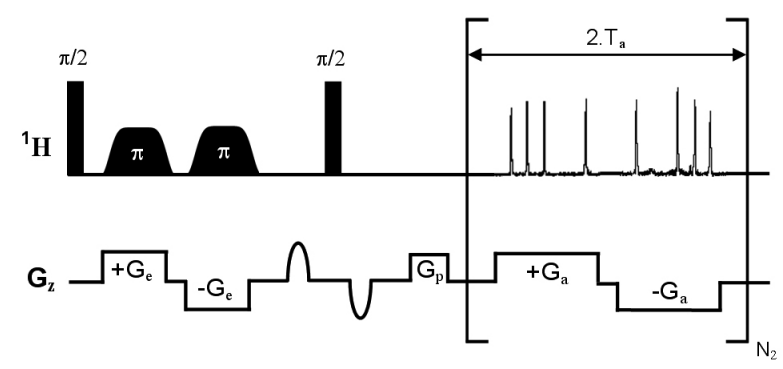

Figure 4S. Ultrafast COSY pulse sequence based on the constant-time spatial encoding scheme proposed by Pelupessy. ${ }^{20}$ To perform this experiment, the coherence selection gradients strength was $45 \mathrm{G} / \mathrm{cm}$ during $1000 \mu \mathrm{s}$, and the purge gradient strength was $-10 \mathrm{G} / \mathrm{cm}$ with $400 \mu$ s of duration. The acquisition times for direct and indirect dimensions were 65.59 and $0.12 \mathrm{~ms}$, respectively. For the acquisition 35 and $-35.008 \mathrm{G} / \mathrm{cm}$ (to compensate for shearing effects) gradients were applied for $236 \mu$ s each, with a gradient rise time of $20 \mu \mathrm{s}$ (recovery delay in pulse sequence) 


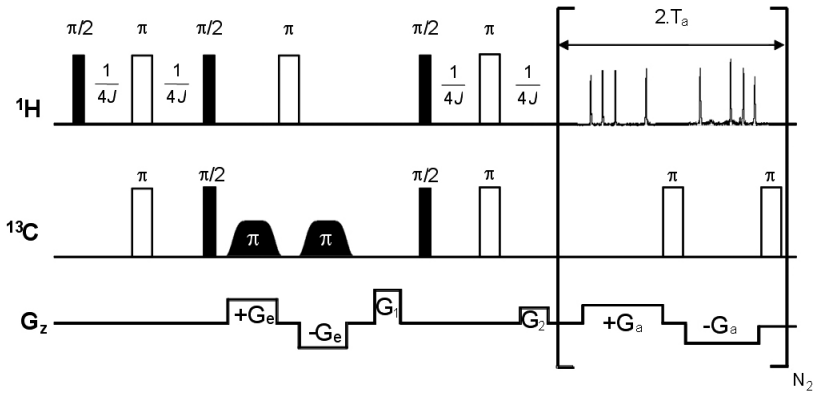

Figure 5S. Ultrafast HSQC pulse sequence based on the constant-time spatial encoding scheme proposed by Pelupessy. ${ }^{20}$ An asymmetric phase cycle $(x,-x, x, x)$ was used within each single shot acquisition on the decoupling pulse to avoid the formation of decoupling artifacts. It was also used a real phase cycle $(x,-x)$, requiring several scans, on the last ${ }^{13} \mathrm{C} 90^{\circ}$ pulse and the receiver. To perform this experiment, identical chirp pulses than those for UF-COSY were used, but applied in the presence of $\pm 20 \mathrm{G} / \mathrm{cm}$ encoding gradients to account for the larger ${ }^{13} \mathrm{C}$ frequency range. The acquisition times for direct and indirect dimensions were 65.59 and $0.02 \mathrm{~ms}$, respectively. Acquisition gradients were identical to those employed for UF-COSY. The INEPT delay was set to $1.72 \mathrm{~ms}$. Eight scans were recorded for sensitivity and phase-cycling purposes

\section{PULSE SEQUENCE FOR UF-COSY}

;ufcosy

;avance-version

; $\$$ CLASS=HighRes

;\$DIM=1D

;\$TYPE=

;\$SUBTYPE=

;\$COMMENT=

\#include <Avance.incl $>$

\#include $<$ Grad.incl $>$

\#include $<$ De.incl $>$

1 ze

100u UNBLKGRAD

$2 \mathrm{~d} 1 \mathrm{pl1}: \mathrm{f} 1$

p1 ph1 $\longrightarrow 90^{\circ}$ pulse

10u gron0

p11:sp1:f1 ph2

$10 \mathrm{u}$ groff

10u gron1

p11:sp1:f1 ph4

10u groff

10u pl1:f1

$10 \mathrm{u}$

p23:gp23 $\longrightarrow$ coherence selection gradient

$10 \mathrm{u}$

p1 ph1

$\longrightarrow \quad$ mixing period

$10 \mathrm{u}$

p26:gp26 $\longrightarrow$ coherence selection gradient

$\mathrm{d} 25$ gron25 $\longrightarrow$ purge gradient

$10 \mathrm{u}$ groff

$10 \mathrm{u}$

ACQ_START(ph30,ph31) $\}$ starting acquisition 1u DWELL_GEN:f1

$3 \mathrm{~d} 20$ gron2 $\longrightarrow$ positive acquisition gradient

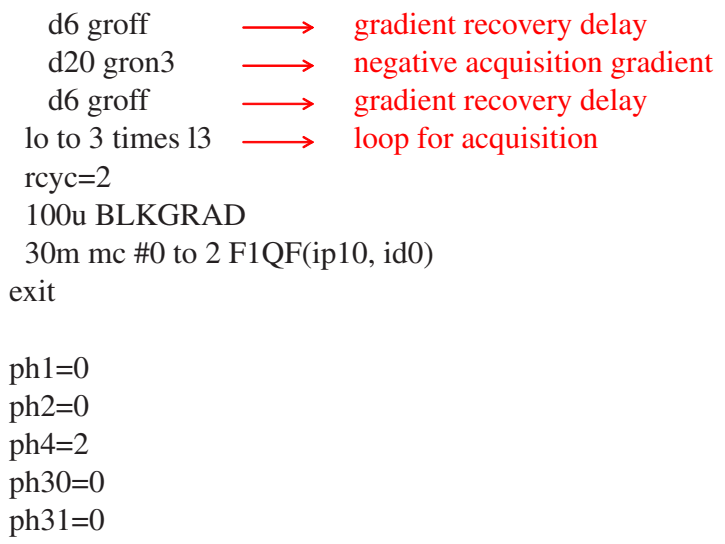

\section{PULSE SEQUENCE FOR UF-HSQC}

;ufhsqc

;avance-version

\section{;\$CLASS=HighRes \\ ; $\$ \mathrm{DIM}=2 \mathrm{D}$ \\ ;\$TYPE= \\ ;\$SUBTYPE= \\ ;\$COMMENT=}

\#include $<$ Avance.incl $>$

\#include $<$ Grad.incl $>$

\#include $<$ De.incl $>$

“p2=p1*2”

"p4=p3*2"

"d $4=1 \mathrm{~s} /(\mathrm{cnst} 2 * 4)$ "

"d6=d4-d15"

"d $10=\mathrm{p} 20 "$

"d11=p21"

"p15=(td*dw)/(2*13)-2*d17-p4"

1 ze

100u UNBLKGRAD

$230 \mathrm{~m} \mathrm{pl2:f2}$

d1 pl1:f1

p1 ph0

d4 (center (p2 ph1) (p4 ph4):f2 )

d4

$\mathrm{p} 1 \mathrm{ph} 2$

(p3 ph3):f2

d11

10u gron0

$\mathrm{p} 7: \mathrm{sp} 1: \mathrm{f} 2 \mathrm{ph} 1$

10u groff

d 10

10u

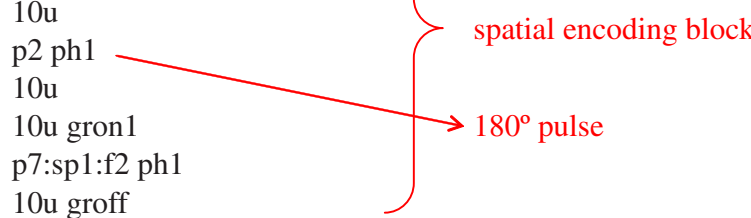

10u groff

$\mathrm{d} 14$ gron $4 \mathrm{pl} 2 \mathrm{f} 2 \longrightarrow$ coherence selection and folding gradient $10 \mathrm{u}$ groff 


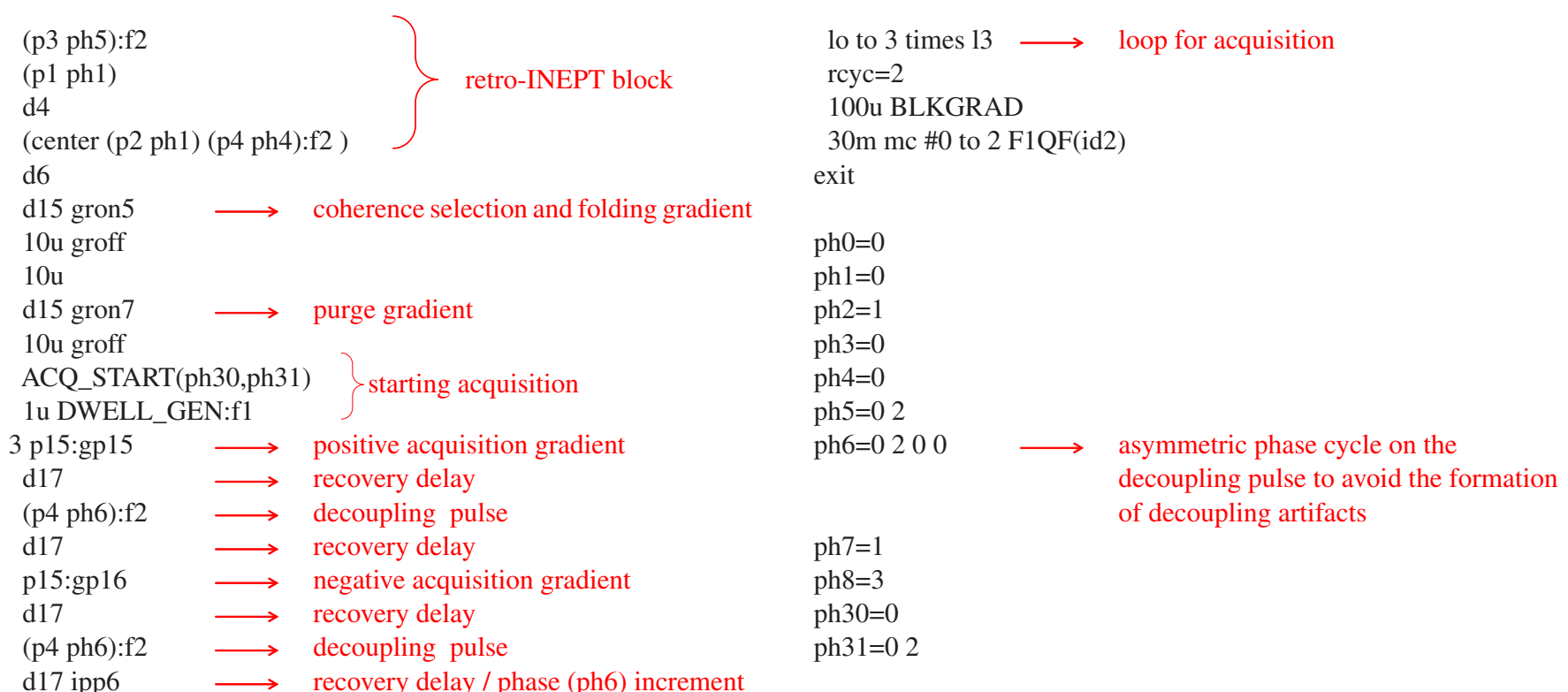

